\title{
Detecting Electronic States at Stacking Faults in Magnetic Thin Films by Tunneling Spectroscopy
}

\author{
A.L. Vázquez de Parga ${ }^{a *}$, F.J. García-Vidal ${ }^{b}$ and R. Miranda ${ }^{a}$ \\ a Departamento de Física de la Materia Condensada and Instituto de Ciencia de Materiales "Nicolás Cabrera" \\ Universidad Autónoma de Madrid. 28049 Madrid. Spain. \\ ${ }^{b}$ Departamento de Física Teórica de la Materia Condensada \\ Universidad Autónoma de Madrid. 28049 Madrid. Spain.
}

(November 1, 2018)

\begin{abstract}
Co islands grown on $\mathrm{Cu}(111)$ with a stacking fault at the interface present a conductance in the empty electronic states larger than the Co islands that follow the stacking sequence of the $\mathrm{Cu}$ substrate. Electrons can be more easily injected into these faulted interfaces, providing a way to enhance transmission in future spintronic devices. The electronic states associated to the stacking fault are visualized by tunneling spectroscopy and its origin is identified by band structure calculations.
\end{abstract}

PACS numbers : 73.20.-r, 61.16.Ch, 73.20.At

The new field of spin electronics or "spintronics" refers to electronic devices in which operation the electron spin is just as important as its charge. For example, the twocomponent intrinsic nature of spintronic devices might allow quantum computing in the solid state. Recently an increasing number of devices that exploit spin-polarized transport (reading heads, sensors, magnetic random access memories, metal base or semiconductor field-effect transistors) have been proposed or built [1]. Some of them take advantage of physical effects, such as the Giant Magnetoresistence (GMR) [2] or the Tunneling Magnetoresistence (TMR) [3], which depend on the spindependent flow of electrons with energies at the Fermi level. Others, such as the spin-valve transistor (SVT) [1], rely on the ballistic transport of hot electrons with energies $0.5-2 \mathrm{eV}$ above the Fermi level.

All these devices contain ferromagnetic/non ferromagnetic interfaces. For instance, the base region of the first spin transistors is formed by a $\mathrm{Co} / \mathrm{Cu}$ multilayer 4 , 5 . The efficient transport of electrons across these $\mathrm{Co} / \mathrm{Cu}$ interfaces is crucial to the behavior of the device. In fact, a severe drawback from the reported spin transistors is the extremely low transmission of electrons with $0.5-2$ $\mathrm{eV}$ above the Fermi level, that result in collector currents ranging from $0.05 \%$ 沟 to $0.004 \%$ [5] of the emitter current.

We report in this Letter an experimental method to characterize locally the transport of electrons at $\mathrm{Co} / \mathrm{Cu}$ (111) interfaces based on Scanning Tunneling Spectroscopy (STS). The method allows us to identify the electronic states related to certain structural arrangement, namely a stacking fault at the $\mathrm{Co} / \mathrm{Cu}$ interface, that enhance the transmission of electrons in the range 0.5-1.5 eV above the Fermi level. The origin of the electronic states is traced with the help of the calculated band structure. These results suggest a way to manipulate the structure of these layered structures that might result in further controlled enhancement of the hot electron transport via properly tuned electronic states.
The experiments were carried out in a UHV chamber with a home made STM, a rear-view LEED optics and Ultraviolet Photoelectron Spectroscopy (UPS). The $\mathrm{Cu}(111)$ substrate was electrochemically polished, sputter-annealed $(500 \mathrm{eV}, 800 \mathrm{~K})$ for 5 minutes and cooled to $300 \mathrm{~K}$. The STM images (Fig. 1(a)) show straight monoatomic steps and no traces of contaminants. The Co film was deposited at room temperature from an electron bombardment source with an evaporation rate of 0.4 ML per minute. The STM tips were made of polycrystalline $\mathrm{W}$ wire electrochemically etched. In order to perform the spectroscopic measurements the tip was cleaned in situ by field emission as described elsewhere [6].

Figure 1(b) shows a topographic $\mathrm{z}(\mathrm{x}, \mathrm{y})$ image of the surface after evaporation of $0.4 \mathrm{ML}$ of Co at room temperature on $\mathrm{Cu}(111)$. In agreement with previous reports [7], there are islands of bilayer $(4.2 \AA)$ height over the $\mathrm{Cu}(111)$ surface, which contain the deposited Co. Recently it has been proposed that the islands are, in fact, three Cobalt layers deep, with the third layer embedded within the $\mathrm{Cu}$ surface layer [8], as schematically depicted in Fig. 1(c). The islands have triangular shape with two different orientations, but, otherwise they appear identical in topographic images. It was suggested [7] that the shape and orientation of the Co islands could be rationalized by considering that they nucleate respectively on the two different threefold absorption sites of the surface as shown in the Fig. 1(c) and that one type of compact step from the two possible on $\mathrm{Cu}(111)$ surfaces, is kinetically favored. This would imply that one of the orientations contains a stacking fault at the interface. Although at this low coverage, diffraction techniques lack enough spatial sensitivity to decide it, a recent quantitative LEED study for a complete bilayer of $\mathrm{Co} / \mathrm{Cu}(111)$ [9] concludes that $15 \%$ of the Co layer presents a stacking-fault at the interface, while the rest of the bilayer follows the FCC stacking sequence of the substrate. Accordingly, the minority islands (pointing to the right in the Figure 1b) would form a twin FCC structure and have a stacking- 
fault (faulted islands), while the majority islands (pointing to the left) would simply continue the FCC arrangement of the $\mathrm{Cu}$ substrate (unfaulted islands).

The lower panel of Fig. 2 shows two differential tunneling conductance images at different bias voltage, sampling the occupied (Fig. 2(b)) and empty electronic states of the sample (Fig 2(c)), respectively. The spectroscopic images have been measured simultaneously with the constant-current topograph shown in Fig. 2(a). From the images is clear that the tunneling conductance for both type of islands is the same at negative sample bias voltage, while it is clearly different at positive sample bias voltage, with the faulted islands pointing to the right showing larger conductance.

$\mathrm{I}-\mathrm{V}$ curves were recorded on top of each type of islands to get a more detailed information about such a prominent spectroscopic feature. The experimental procedure was as follows. First an I-V curve was measured on the substrate to verify that the tip was clean, stable and reproduced the spectrum of the well known surface state of $\mathrm{Cu}(111)$ 10]. Then we recorded I-V curves on top of one type of Co island, checked that the tip did not change by recording another spectrum on clean $\mathrm{Cu}(111)$ (between the islands) and afterwards we measured on the other type of Co island. In this way, differences in the spectra can be assigned to changes in the electronic structure of the islands and not to uncontrolled changes in the tip structure that often produce localized electronic states in the tip apex which show up in the I-V curves [6]. Figure $3(\mathrm{a})$ shows the characteristic surface state peak of clean $\mathrm{Cu}(111)$ at $-0.4 \mathrm{~V}$ on the clean patches of the surface and a rather featureless tunneling spectra on both types of Co islands. The spectra on the islands are identical for negative sample bias (occupied states) but different for positive sample bias (empty states). In particular the minority (faulted) islands present higher conductance for positive bias voltage in agreement with Fig. 2 .

In order to verify if a fault in the stacking sequence can provoke such a dramatic change in the tunneling conductance of the islands, band structure calculations were carried out using a simple tight-binding formalism. In our theoretical framework, islands are modeled by a laterally-infinite triple overlayer of Co joined to a 20-layer $\mathrm{Cu}$ (111) film. This is reasonable since the lateral size of the islands is large $(100 \AA)$ and the spectra are taken in the center of the islands. Hopping tight-binding elements for the $\mathrm{Cu}-\mathrm{Cu}$ and $\mathrm{Co}-\mathrm{Co}$ bonds are taken from Ref. 11] whereas we approximate the hopping $\mathrm{Cu}-\mathrm{Co}$ by the average of $\mathrm{Cu}-\mathrm{Cu}$ and $\mathrm{Co}-\mathrm{Co}$ interactions [12]. For the $\mathrm{Co}-\mathrm{Co}$ interactions we have used the spin-polarized parameters. We have not included atomic rearrangement in our calculations: this effect is known to be small in metallic systems where atomic density and local environment are preserved in the presence of a stacking-fault. We have calculated the band structure of the slab for two different kinds of stacking sequence of the cobalt overlayer
(ABCabc and ABCacb). As the energy levels and hopping elements are exactly the same, the structural arrangement is therefore the only difference between the two cases.

The results of our calculation are shown in Fig. 3(b) and 3(c), where the band structure of the slab is projected on the surface Brillouin Zone and displayed along the $\bar{\Gamma}-\overline{\mathrm{M}}$ direction for the two kind of stacking sequences: unfaulted in (b) and faulted with the fault located between the first and second Co layers in (c). In the $\bar{\Gamma}-\overline{\mathrm{K}}$ direction the results of our calculation are similar (not shown). First it is interesting to point out that states that change depending on the stacking sequence appear within the $\mathrm{Cu}(111)$ gap region 13]. Above the Fermi level (located at $\mathrm{E}=0$ in the figures), the band structure of the slab is governed by six bands that have mainly $d_{x z}$ and $d_{y z}$ character ( $z$ is normal to the surface). The bands 5 and 6 have very low amplitude at the surface. Bands 1 to 4 , on the contrary, have strong weight at the surface and are sensitive to the presence of a stacking-fault in the Co-trilayer. The stacking fault breaks the symmetry in the xy-plane and the resulting different hybridization between the $d_{x z}-d_{y z}$ orbitals of the Co layer (that are effectively decoupled of the $\mathrm{Cu}(111)$ bulk states) changes the dispersion relation of these bands and provokes the splitting of them at the $\Gamma$ point as seen in Fig. 3. This distinct dispersion with parallel momentum is a signal of a different spatial distribution of the electronic states associated with these bands in the two island orientations. For the faulted islands the first four bands are remarkably flatter which implies that the corresponding wavefunctions are more delocalized in the z-direction (and then extending more into vacuum) that their counterparts in the unfaulted islands. As a way of example, the Local Density of States (LDOS) at $5 \AA$ above the surface associated with bands $3-4$ at $\Gamma$ point is 1.5 times greater in the faulted islands than in the unfaulted ones. Then, it is expected to obtain larger tunneling probabilities and hence larger conductance when the tip is placed above a faulted island that when it is above an unfaulted one. And this, over a large energy range due to the splitting of the bands.

These results indicate that the structural arrangement right at $\mathrm{Co} / \mathrm{Cu}(111)$ interfaces determines the electronic states and the conductance across such interfaces. This opens the possibility to enhance hot-electron transport across $\mathrm{Co} / \mathrm{Cu}$ multilayers by properly engineering the geometric and electronic structure of the Co layers.

In order to explore this possibility, we take advantage of features that develop spontaneously during the growth of $\mathrm{Co}$ on $\mathrm{Cu}(111)$. Although the majority islands of $\mathrm{Co}$ continue the FCC stacking sequence of the $\mathrm{Cu}$ substrate upon further Co deposition, the film quickly starts to adopt the HCP, bulk-like structure [14]. In effect, Co atoms in the third layer on choose between the two threefold adsorption sites with almost equal probability giving 
rise to a pyramidal-shaped morphology [7], with pyramids presenting the FCC or HCP stacking sequence as deduced from surface X-ray diffraction data [14]. We show below that their different electronic structure can be locally identified by STS.

For tips showing a strong $\mathrm{Cu}(111)$ surface state bandedge, only two predominant types of spectra were observed at different points on $6 \mathrm{ML}$ thick Co films. These are shown in Fig. 4(a) and (b). Similarly to the initial islands, the conductance corresponding to the occupied states is almost identical, but clear differences are seen in the empty states. In panels (c) and (d) of the same figure, we present the calculated total LDOS around $\bar{\Gamma}$ for a Cobalt film $6 \mathrm{ML}$ thick with FCC and HCP stacking sequences, respectively, at different distances from the surface. By simply comparing Figs. 4c-d with Figs. 4ab, we can conclude that pyramids showing spectra like (a) follow a FCC stacking whereas spectra like (b) are linked to HCP stacking. Noting that the FCC phase shows a larger LDOS than the HCP phase, we could suggest that varying growth conditions (e.g. the use of surfactants, coevaporation or pulsed laser deposition) that could stabilized the FCC rather than the HCP phase of Co might yield new ways to enhance the transmission in $\mathrm{Co} / \mathrm{Cu}$ multilayers.

The physical origin of the differences between the electronic structure of both stacking sequences is the same than for the thinner islands. When the stacking sequence is FCC, bands associated to $\mathrm{d}_{x z}-\mathrm{d}_{y z}$ orbitals of the Co layer are degenerated at the $\Gamma$ point in a way very much similar to the case of unfaulted islands (see Fig. 3b). Hence a peak in the LDOS at $0.5 \mathrm{eV}$ above Fermi level is expected (see Fig. 4c). However when the stacking sequence is HCP, the different hybridization of those orbitals provokes the opening of a gap at the $\Gamma$ point also in accordance of the case of faulted islands (see Fig. 3c). Therefore a LDOS with two peaks located below and above $0.5 \mathrm{eV}$ is obtained as seen in Fig. 4d. The agreement between the experimental data and the calculated density of states is remarkable. Even the relative intensity between the FCC and HCP films in the calculations and experiments is conserved.

In conclusion, we have found that the differential tunneling conductance is higher on Cobalt islands grown on $\mathrm{Cu}(111)$ that have a stacking fault at the interface than on islands that continue the FCC-stacking of the substrate. Using a simple theoretical model, in which the stacking sequence is the only difference between both type of islands, we have identified the electronic states responsible for the enhanced conductance. These results open a possibility to enhanced the transmission of hot electrons across magnetic/non magnetic interfaces by properly tuning the geometric and electronic structure of these artificial materials.

This work has been financed by grants MAT98-C04-02 and PB97-0031.
* e-mail:al.vazquezdeparga@uam.es

[1] G.A. Prinz, Science 282, 1660 (1998).

[2] M.N. Baibich et al., Phys. Rev. Lett. 61, 2472 (1988).

[3] J.S. Modera et al., Phys. Rev. Lett. 74, 3273 (1995).

[4] D.J. Monsma et al., Phys. Rev. Lett. 74, 5260 (1995).

[5] D.J. Monsma et al., Science 281, 407 (1998).

[6] A.L. Vázquez de Parga et al., Phys. Rev. Lett. 80, 357 (1998).

[7] J. de la Figuera et al., Phys. Rev. B 47,13043 (1993).

[8] M.O. Pedersen et al., Surf. Sci. 387, 86 (1997).

[9] Ch. Rath et al., Phys. Rev B 55, 10791 (1997).

[10] M.F. Crommie et al., Nature 363, 524 (1993).

[11] D.A. Papaconstantopoulos, Handbook of the band structure of elemental solids, Plenum Press, New York (1986).

[12] J. Izquierdo et al., Phys. Rev. B 55, 445 (1997).

[13] The first calculations reporting the existence of occupied electronic states of d-character associated to stacking faults were performed on $\mathrm{Ni}(111)$, see F. Yndurain and L.M. Falicov, Phys. Rev. Lett. 37, 928 (1976)

[14] J. Camarero et al., Mat. Res. Soc. Symp. Proc. 384, 49 (1995).

FIG. 1. (a) $(1000 \times 1000 \AA)$ STM image of the clean $\mathrm{Cu}(111)$ surface. (b) $(1500 \times 1500 \AA) \quad 0.6 \mathrm{ML}$ of Cobalt deposited at room temperature with an evaporation rate of $0.4 \mathrm{ML}$ per minute. Two types of bilayer-high (4.2 A) triangular islands appear on the surface. The islands are marked with "u" (unfaulted) and "f" (faulted) depending on the stacking sequence. (c) Model proposed for the two types of islands shown in panel (b). The islands are three layers high with one layer within the copper surface. The unfaulted ("u") islands follow the FCC stacking sequence and the faulted ones ("f") present an stacking-fault.

FIG. 2. (a) $(500 \times 500 \AA)$ STM image after deposition of 0.4 $\mathrm{ML}$ of Cobalt on $\mathrm{Cu}(111)$ at room temperature. There is no difference in the height of both type of islands in the topographic images within the noise. Panels (b) and (c) shown spectroscopic images, $(\mathrm{dI} / \mathrm{dV})(\mathrm{x}, \mathrm{y})$, of the region shown in (a), measured with the feedback loop stabilized at a sample bias of (b) $-1.5 \mathrm{~V}$ and (c) $+1.5 \mathrm{~V}$. The bias was modulated $(70 \mathrm{meV})$ with a frequency $(8 \mathrm{kHz})$ higher than the feedback loop response and the ac-component of the current was measured by a lock-in detector. Bright areas correspond to high differential conductance.

FIG. 3. (a) Experimental differential conductance for a clean area of the $\mathrm{Cu}(111)$ surface (solid line), for a Co island that follows the $\mathrm{Cu}$ stacking (unfaulted) (dashed line) and for a Co island with a stacking fault (faulted) (dotted line). Energy bands in the [111] direction for (b) the unfaulted Co island and (c) for the island that presents a stacking-fault at the interface. 
FIG. 4. Panels (a) and (b) present tunneling spectra measured over different places in a 6 ML-thick film of Co grown on $\mathrm{Cu}(111)$ at room temperature. Panels (c) and (d) display the calculated LDOS at different distances from the surface, integrated over $\mathrm{k}_{\|}$in a circular region around $\bar{\Gamma}$, out to $\frac{1}{4}$ of the $\bar{\Gamma}-\bar{M}$ distance, for $6 \mathrm{ML}$ of Co following (c) an FCC stacking sequence and (d) an HCP stacking one. The prominent peaks at $\sim 0.5 \mathrm{eV}$ correspond to unoccupied minority electron states. The calculated LDOS has been smoothed with a Gaussian of 0.2 eV FWHM to simulate experimental resolution. 


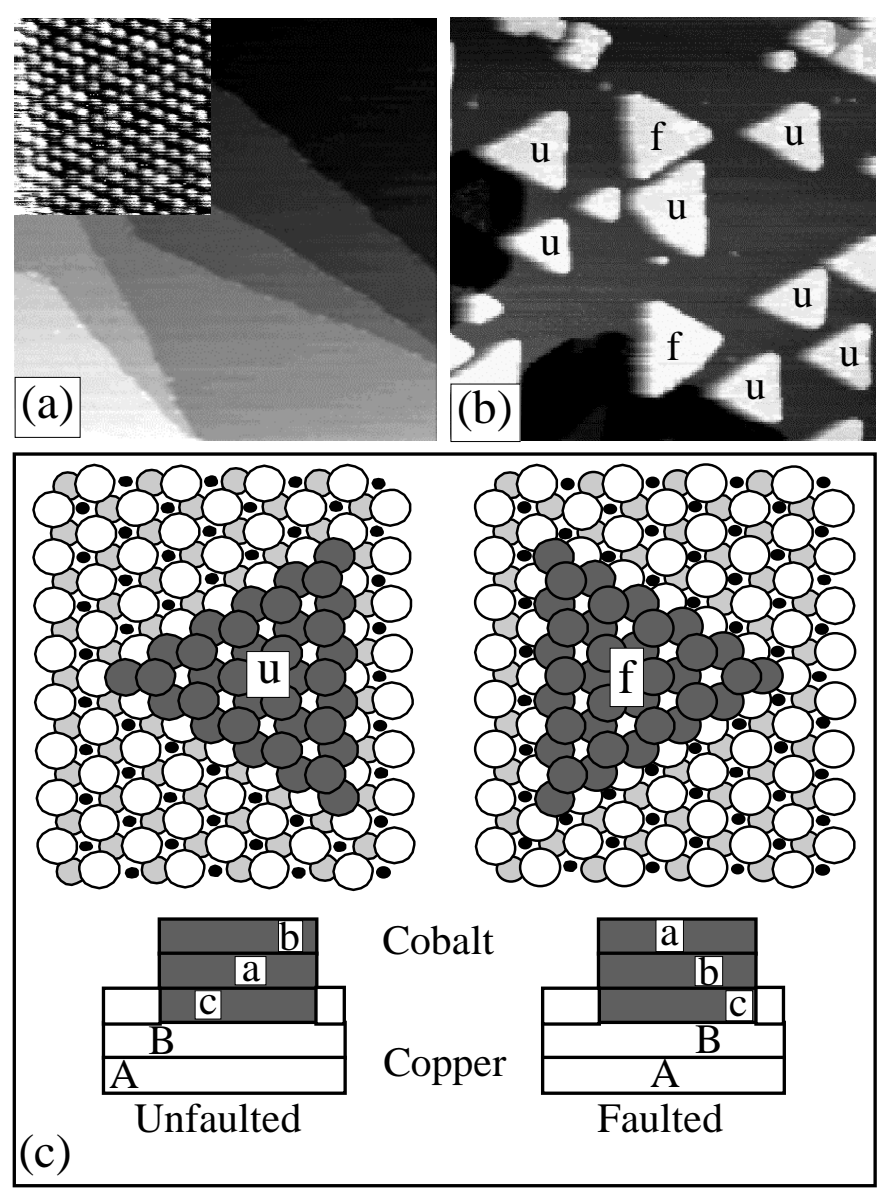



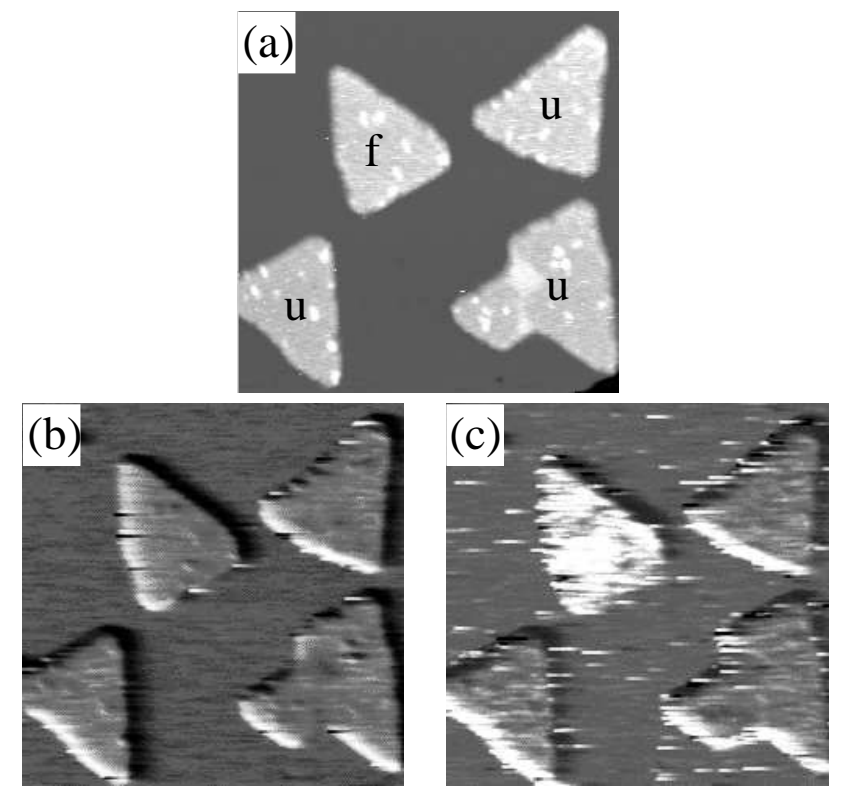

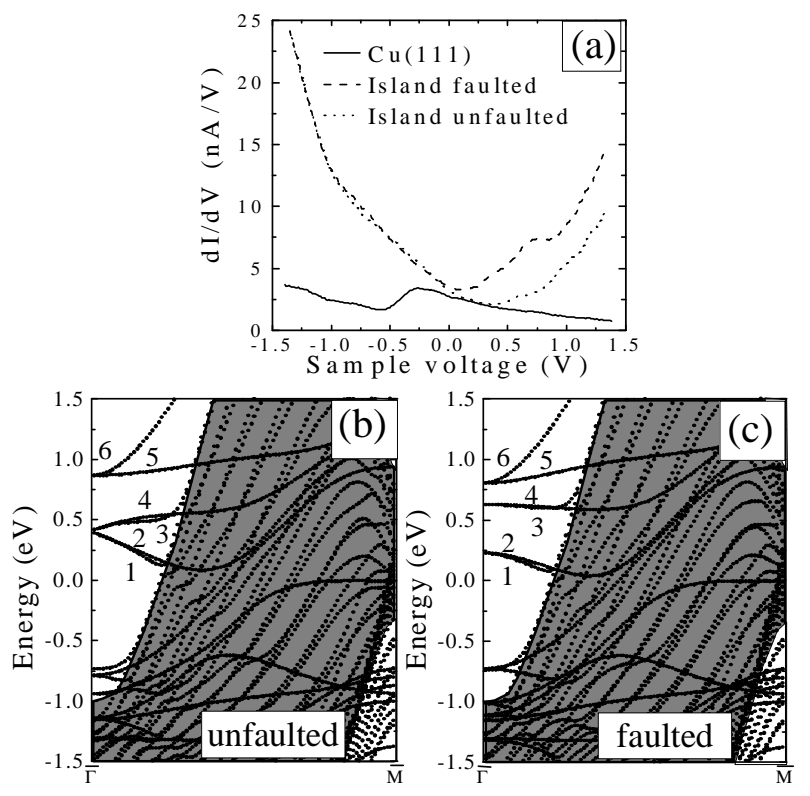

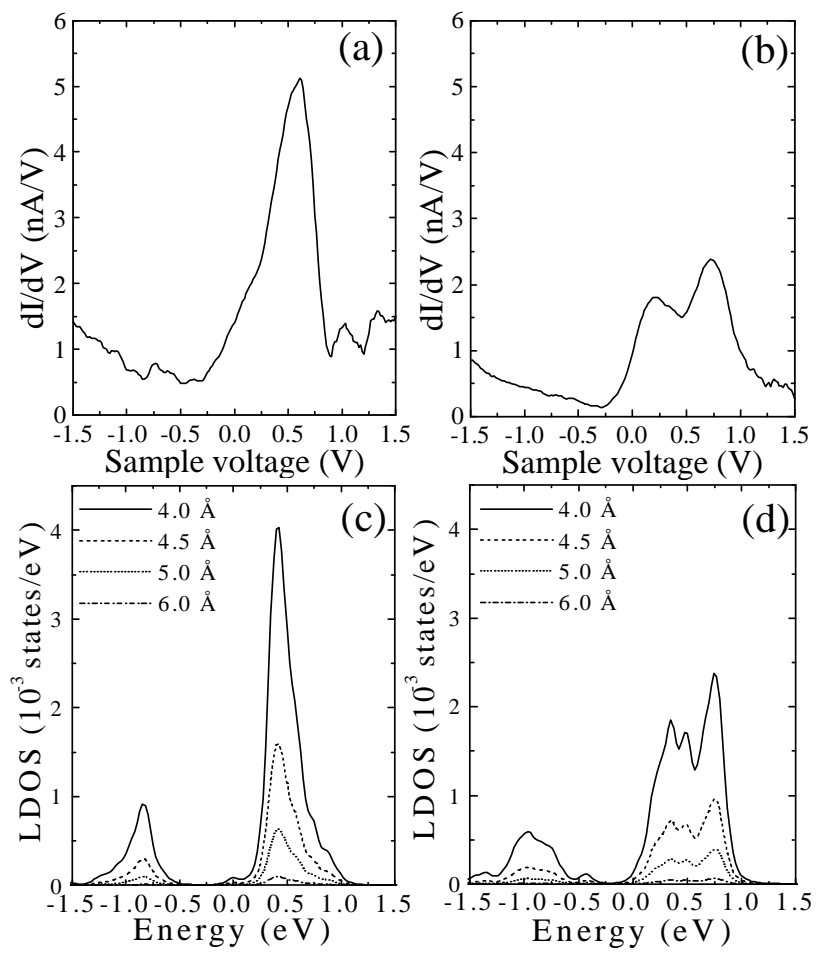